\title{
Desarrollo de una metodología para la formación y evaluación “continua” en pensamiento crítico
}

\author{
Santiago Vidal-Puiga, Susana Barceló-Cerdáb, Ana Debón ${ }^{c}$, Ángeles Calduch-Losad y \\ Fulgencia Villa \\ Departamento de Estadística e Investigación Operativa y Calidad - UPV

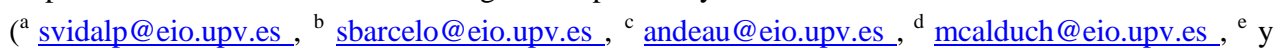 \\ mfuvilju@eio.upv.es)
}

\begin{abstract}
In this study, we shall describe a teaching experience carried out by a group of lecturers in the Statistics Department. These lecturers are involved in the training and assessment of "critical thinking" skills as part of the institutional project centred on generic skills at the Universitat Politècnica de València.

The objective of this experience was to promote the understanding, development and application of the critical thinking skills in the students. Different activities linked to the abilities involved in critical thinking are proposed to the students throughout the academic year. The activities require ongoing assessment from the formative point of view. Thus, the students are aware of their own progress. Additionally, students are evaluated in the exams with questions in different formats (PoliformaT exams, tests and peer review).

Suitable materials and activities for training and assessment of critical thinking have been developed. Finally the teaching staff and the students' opinions have been collected through surveys.
\end{abstract}

\section{Keywords:}

Keywords: Critical thinking, ongoing assessment, generic skills

\footnotetext{
Resumen

En este trabajo se presenta la experiencia realizada por un grupo de profesores del Departamento de Estadística en diversas asignaturas que son punto de control de la competencia "Pensamiento Crítico". El objetivo de la experiencia era guiar a los alumnos para que entendiesen el pensamiento crítico, lo desarrollasen y lo aplicasen. Durante el curso los alumnos han realizado actividades que trabajan diferentes habilidades vinculadas con dicha competencia. Estas actividades han sido evaluadas de tal manera que desde el punto de vista formativo, el alumno sea consciente de su progreso en la competencia. Adicionalmente se les ha evaluado en los exámenes con cuestiones bajo formatos diversos (exámenes Poliformat, preguntas en pruebas escritas y corrección por pares). Se han creado materiales y
} 
actividades adaptadas para la formación y evaluación del pensamiento crítico y a través de encuestas se ha constatado que el profesorado y el alumnado ha valorado positivamente la metodología empleada.

Palabras clave: Pensamiento crítico, evaluación continua, competencia transversal.

\section{Introducción}

De acuerdo con el nuevo proyecto institucional en competencias transversales de la Universitat Politècnica de València (UPV), los títulos de grado y posgrado han de incorporar la formación de los estudiantes en competencias transversales o genéricas, así como la evaluación de su nivel de logro. En este trabajo se explica cómo hemos enfocado y gestionado la incorporación en nuestras disciplinas de la formación y evaluación del alumnado en pensamiento crítico, tanto en las clases de teoría como en las sesiones prácticas y mediante evaluación continua. Cabe destacar que para el presente trabajo solicitamos a la UPV y se nos concedió un Proyecto de Innovación y Mejora Educativa (PIME Referencia_B10 del 2016-2017). Este proyecto se ha centrado en las asignaturas de estadística de 4 titulaciones de la Escuela Técnica Superior de la Ingeniería de Diseño (ETSID) y 3 titulaciones de la Escuela Técnica Superior de Ingeniería Agronómica y del Medio Natural (ETSIAMN) en la UPV. El número de alumnos implicados está próximo a 600 y la mayoría de las asignaturas involucradas se encuentran dentro de los dos primeros años de las titulaciones de grado envueltas.

La UPV, dentro de su proyecto institucional en competencias transversales establece 3 niveles de dominio para cada competencia transversal con el objetivo de que la adquisición y mejora en cada competencia sea progresiva a lo largo de las titulaciones. De este modo, en las asignaturas de primeros cursos de Grado se sugiere centrarse en el nivel de dominio I, con el resultado de aprendizaje, de acuerdo a lo establecido por la UPV en relación a la competencia de pensamiento crítico, de mostrar una actitud crítica ante la realidad, siendo capaz de analizar y cuestionar información, resultados, conclusiones y otros puntos de vista.

Nuestro grupo, formado por profesores del Departamento de Estadística e Investigación Operativa Aplicadas y Calidad, decidió que la manera más eficiente de colaborar en dicho proyecto era especializarnos en una de las competencias transversales para constituirnos en uno de sus puntos de control. Escogimos el pensamiento crítico por tratarse de una competencia instrumental directamente relacionada con el pensamiento estadístico (Aizikovitsh-Udi et al., 2016) indispensable para el análisis de datos y, consecuentemente, toma de decisiones de una forma objetiva y razonada. Además, siendo conscientes de que enseñar a pensar críticamente requiere mucho tiempo y mucha práctica reflexiva centrada en una amplia variedad de ejemplos, nuestro grupo decidió apostar por alguna metodología que ponga al alumno en contacto con múltiples y diversas situaciones donde las habilidades y disposiciones asociadas al pensamiento crítico se estimulen y desarrollen. Para ello, se incluyeron en nuestras sesiones de prácticas de laboratorio y en las prácticas de aula diferentes ejercicios y actividades con incidencia en el pensamiento crítico.

(cc) EY-NC-ND 2018, Universitat Politècnica de València

Congreso IN-RED (2018) 
Respecto a las distintas formas de evaluación de dicha competencia transversal, una de las cosas que más llama la atención es la falta de existencia de pruebas o test que sirvan para la evaluación del pensamiento crítico en materias específicas y, por tanto, centradas en determinadas áreas de conocimiento. Además, el pensamiento crítico es una competencia que incluye muchas y variadas habilidades y por tanto, la dificultad estriba en poder encontrar qué tipo de pruebas pueden ser las más adecuadas para formar y evaluar a los alumnos en las mismas. Para ello, aprovechando las posibilidades actuales de la plataforma educativa de la UPV, hemos decidido hacer uso de una estrategia mixta para la evaluación que utilice pruebas de tipo test opción múltiple en sus distintas modalidades (Norris y Ennis 1989) y pruebas tipo ensayo o informe de acuerdo con las habilidades o disposiciones consideradas en cada caso, siendo este último tipo de prueba el más apropiado para la evaluación de las habilidades más creativas asociadas al pensamiento crítico.

Por último, indicar que nuestro equipo considera como aspecto muy importante el que el profesorado implicado pueda alcanzar una adecuada preparación que les permita abordar la formación y evaluación de los alumnos en dicha competencia con las metodologías y herramientas más adecuadas. Agradecemos y consideramos relevante el esfuerzo realizado por el ICE (Instituto de Ciencias de la Educación de la UPV) en su ayuda de formación al profesorado.

\section{Objetivos}

De acuerdo con lo expuesto en el apartado de introducción y en concordancia con los objetivos que se ha impuesto la UPV, nuestro grupo se unió al proyecto de formación del alumnado en competencias transversales, y lo convirtió en un objetivo prioritario.

El objetivo general del presente proyecto es el desarrollo de una metodología para la formación y evaluación "continua” en pensamiento crítico en las asignaturas de Estadística de distintas titulaciones.

Dicho objetivo ha supuesto:

A) El estudio profundo de la competencia y la adopción de una definición de pensamiento crítico acorde con la asignatura de Estadística.

B) La elaboración de material y preparación de actividades contextualizadas en temas relacionados con nuestra materia específica que sean adecuados para la formación y evaluación de los alumnos en pensamiento crítico.

C) La implantación de una metodología de evaluación continua y formativa en la misma.

Dentro de los objetivos especificos cabría citar los siguientes:

1) Preparar material de información sobre la competencia transversal:

El alumno debe saber en qué consiste la competencia en la que se le pretende formar y evaluar. 
2) Preparar material de formación en pensamiento crítico para incluir en los guiones de prácticas:

- Preguntas con respuesta abierta o actividades donde se tratarán cuestiones de estadística que incorporen aspectos del pensamiento crítico.

- Resoluciones detalladas y comentadas desde el punto de vista estadístico y de las diferentes habilidades del pensamiento crítico consideradas.

- Otras posibles actividades en las que dentro de un contexto estadístico se trate de formar al alumno en pensamiento crítico.

3) Preparar preguntas para la evaluación continua en pensamiento crítico durante las sesiones de prácticas:

Junto a las posibles formas de evaluación de los conocimientos y habilidades en la materia de estadística, cada práctica incluirá adicionalmente una parte de evaluación formada por unas pocas preguntas centradas en temas estadísticos, donde se requiera de modo específico el uso de ciertas habilidades del pensamiento crítico. Estas preguntas serán cuestiones de tipo test de opción múltiple y otros formatos soportados por la herramienta exámenes de PoliformaT (plataforma educativa implantada en la UPV).

4) Preparar actividades individuales o grupales para evaluar las habilidades más creativas de pensamiento crítico y donde se puedan evaluar habilidades combinadas:

Los resultados de estas actividades se presentarán en forma de informe. Se considerará la posibilidad de incluir una evaluación entre pares, y la posibilidad de realizar sugerencias de mejoras entre distintos grupos.

5) Creación de pruebas y adaptación de las rúbricas propuestas por el ICE para la evaluación de la competencia en nuestra materia específica:

Las preguntas creadas se incorporarán a distintas pruebas de evaluación y baterías de exámenes de PoliformaT, y se realizará la evaluación final del alumno haciendo uso de las rúbricas propuestas por el ICE, adaptadas a nuestras metodologías y materias impartidas.

6) Hacer un estudio estadístico de los resultados obtenidos que nos permita validar la metodología empleada y nos proporcione posibles sugerencias de mejora.

7) Comunicar y compartir nuestros resultados con la comunidad universitaria haciendo, por tanto, una difusión adecuada de los resultados obtenidos.

\section{Desarrollo de la innovación}

En la innovación presentada se apostó por la implantanción de una metodología de evaluación continua y formativa en la competencia transversal que aumentase el contacto

(cc) EY-NC-ND 2018, Universitat Politècnica de València

Congreso IN-RED (2018) 
del alumno con diferentes situaciones donde se estimulan y desarrollan las habilidades y disposiciones asociadas al pensamiento crítico.

Para ello se han seguido las siguientes fases:

- Fase 1: Definición de pensamiento crítico

Una de los primeras tareas a realizar fue establecer la definición de pensamiento crítico con la que iba a trabajar el grupo. Todas las definiciones encontradas enfatizan la importancia de la claridad de ideas y la racionalidad, y nos inclinamos por la de Ennis (1993) "el pensamiento crítico se define como un pensamiento reflexivo y razonable y con el propósito de que la persona pueda decidir qué creer o hacer”. Ahondando un poco en esta definición, vemos que este pensamiento es reflexivo, porque analiza resultados y situaciones; es razonable, porque predomina la razón sobre otras dimensiones del pensamiento y solo los pensamientos basados en buenas razones conducen a las mejores soluciones; y finalmente tiene un propósito, porque está dirigido conscientemente hacia una evaluación de posibles afirmaciones (qué creer) o acciones (qué hacer). El pensamiento crítico incluye además aspectos creativos como la concepción de alternativas, la formulación de hipótesis y definiciones y el desarrollo de planes experimentales.

Los expertos en el tema han confeccionado una lista muy extensa de las habilidades y disposiciones necesarias para alcanzar un buen pensamiento crítico. A la vista de esta definición y la gran cantidad de habilidades y disposiciones implicadas, se puede apreciar que el pensamiento crítico incluye muchos y variados aspectos, por lo que la formación del alumnado en los mismos y su correcta evaluación se convierte en todo un reto.

El tener una definición de pensamiento crítico era un aspecto importante, dado que se requiere una definición para identificar las habilidades y elementos que son necesarios incorporar en todos y cada uno de los ámbitos (clases de teoría, prácticas, actividades...) que integran las asignaturas a impartir.

- $\quad$ Fase 2: Selección de los indicadores

Como establecimos en la justificación, nuestro ámbito de acción se centra en el nivel I de dominio de la competencia. En este nivel, nuestro grupo ha decidido considerar los indicadores de la tabla 1 para la formación y evaluación de los alumnos en pensamiento crítico. Es importante fijar los indicadores, porque consideramos que esto puede ayudar a determinar los distintos tipos de actividad a realizar y el modo de evaluarlas.

Cabe señalar que en las diferentes asignaturas impartidas por los profesores participantes en el presente PIME se ha hecho uso de materiales y estrategias de evaluación de naturaleza variada, con el objetivo de mejorar nuestro conocimiento sobre el grado de éxito de las distintas opciones planteadas. 
Tabla 1: Indicadores para medir el avance en el pensamiento crítico

\begin{tabular}{ll}
\hline \multicolumn{1}{c}{ Indicadores } \\
\hline $\mathbf{I}_{\mathbf{1}}$ & $\begin{array}{l}\text { Muestra una actitud crítica ante la realidad: se pregunta el porqué } \\
\text { de las cosas. }\end{array}$ \\
$\mathbf{I}_{\mathbf{2}} \quad \begin{array}{l}\text { Elabora un discurso argumentándolo con razones, asunciones y } \\
\text { evidencias. }\end{array}$ \\
$\mathbf{I}_{3} \quad \begin{array}{l}\text { Detecta incoherencias o contradicciones en el discurso de otras } \\
\text { personas o en un texto. }\end{array}$ \\
\hline
\end{tabular}

- Fase 3: Adaptación, creación e implementación de actividades, evaluaciones y materiales expositivos

Se han diseñado diferentes actividades para formar y evaluar, y en alguna asignatura se ha introducido algún tema de actualidad vinculándolo con la asignatura.

Entre las actividades y materiales creados cabe destacar:

- Se han propuesto ejercicios en los que se ha realizado un análisis de datos con técnicas estadísticas correspondientes a un determinado tema y los alumnos lo han examinado, rebatiendo o aceptando con argumentos bien construidos y válidos la información recibida y rehaciendo el análisis e interpretación de resultados hasta llegar a una toma de decisiones adecuada, razonada y bien fundamentada. Este tipo de actividades se evalúan bien por parte del profesor/a, o bien entre compañeros, ya que la plataforma permite la corrección por pares. Se evalúa con una rúbrica, con lo que se realiza una evaluación cualitativa del nivel de logro alcanzado en la competencia. A modo de ejemplo, la Figura 1 presenta una de las actividades utilizadas en una de las asignaturas participantes en el PIME.

- En la actividad descrita se utilizó la correción por pares. Esto nos permitió, por un lado, disponer de la corrección por parte del evaluador de la actividad realizada por el alumno, y por otro, disponer de los comentarios del evaluador que pudieron ser utilizados para calificar el indicador I3 definido en la Tabla 1.

(cc) EY-NC-ND 2018, Universitat Politècnica de València

Congreso IN-RED (2018) 


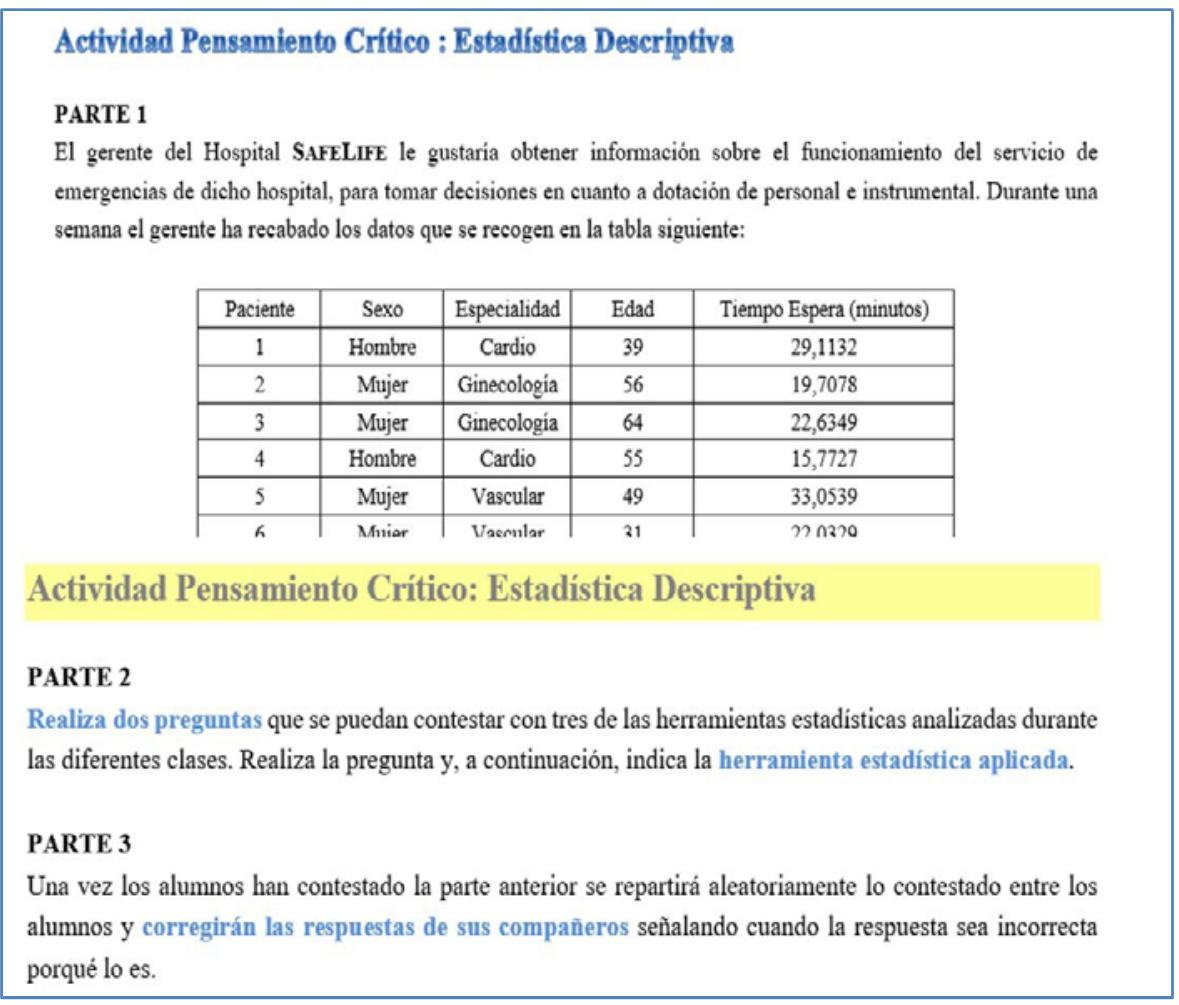

Fig. 1 Ejemplo actividad propuesta

- Pruebas objetivas: Se han realizado usando la plataforma PoliformaT con una batería de preguntas confeccionada con varias cuestiones en las que se presentan al alumno diferentes afirmaciones que pueden o no ser correctas y el alumno tiene que elegir. Estas pruebas las corrige automáticamente la plataforma.

- Pruebas con preguntas de respuesta corta: Se han realizado usando la plataforma PoliformaT y se trata de preguntas donde al alumno se le plantea un problema y diferentes posibles soluciones al mismo. El alumno realiza la correspondiente elección dando una respuesta breve que justifique críticamente su decisión. La corrección la realiza el profesor, pudiendo valorarse cuantitativa o cualitativamente con una rúbrica dependiendo de la naturaleza de la pregunta. Cabe señalar que la plataforma ofrece un entorno cómodo que agiliza y facilita la corrección.

La Figura 2 presenta una de las preguntas de respuesta corta utilizadas en una prueba de una de las asignaturas participantes en el PIME. Puede observarse también que el grupo utilizó un icono para señalar la presencia de las diferentes cuestiones relacionadas con el pensamiento crítico con objeto de que el alumno fuera consciente del carácter especial de esas preguntas. 
Con los datos obtenidos de los 97 pacientes se ha obtenido una tabla de contingencia que se presenta a continuación:

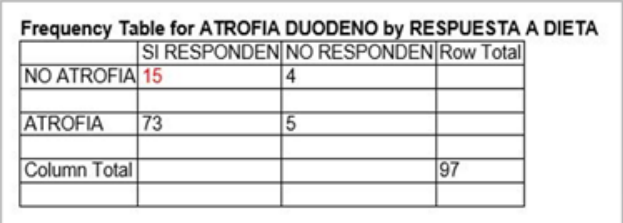

Una doctora investigadora de este estudio, al analizar los resultados de esta tabla concluye que puesto que entre los que no responden a la dieta libre de gluten hay $4 \sin$ atrofia y 5 con atrofia, la atrofia no tiene ninguna relación con la respuesta a la dieta.

- ¿Qué opináis sobre la conclusión de la doctora?

- Dad una argumentación critica y razonada sobre esta conclusión

taitar Pensamiento de Pensamiento Critico (Descriptiva)

pregunta para evaluar la competencia transversal de Pensamiento crítico

Fig. 2 Ejemplo pregunta respuesta corta

- Preguntas en los exámenes escritos: Se preguntan cuestiones o se plantean ejercicios relacionados con las actividades descritas anteriormente que se corrigen por parte del profesor/a y se puntúan numéricamente. Esta puntuación se utiliza tanto para la evaluación de la asignatura propiamente dicha, como para la evaluación de la competencia transversal.

- Tareas basadas en análisis de encuestas relacionadas con problemas contemporáneos. Por ejemplo, en una de las asignaturas, los alumnos han trabajado un tema actual como el de los refugiados como consecuencia de la guerra en Siria como sigue: en primer lugar contestaron a una serie de preguntas donde tenían que señalar su fuente de información. A continuación, tenían que contestar una encuesta relacionada con diferentes preguntas vinculadas con el tema en cuestión. Teniendo en cuenta los datos de la encuesta, aplicaban diferentes técnicas estadísticas para obtener información. Por último, se invitaron a dos chicas voluntarias en campos de refugiados, una de ellas enfermera y que actualmente sigue trabajando en ellos, que contaron su experiencia y cómo funcionan los campos de refugiados. Los alumnos pudieron hacerles preguntas estableciéndose una discusión muy interesante. Tras esta experiencia, los alumnos volvieron a contestar la encuesta inicial y aplicaron de nuevo técnicas estadísticas para obtener información. Esta actividad se llevó a cabo a lo largo del último mes de curso para que se pudieran aplicar las técnicas estadísticas más avanzadas. Además, implicaba trabajar la parte más creativa del pensamiento crítico, ya que la actividad ha estado integrada por varias fases donde la participación del alumno ha sido más activa y menos dirigida por parte del profesor.

Señalar que a modo incipiente y experimental, en uno de los grupos de la asignatura de estadística del grado de Mecánica, se utilizaron las plataformas

(cc) EY-NC-ND 2018, Universitat Politècnica de València

Congreso IN-RED (2018) 
Kahoot y Facebook para que los alumnos contestaran cuestiones de verdadero y falso por un lado y, por otro, comentaran de manera crítica noticias de actualidad relacionadas con la estadística, en las que los alumnos han detectado fallos y han propuesto cómo corregirlos.

- Respecto a las distintas formas de evaluación, una de las cosas que más llama la atención es la carencia de pruebas o test que sirvan para la evaluación del pensamiento crítico en materias específicas $\mathrm{y}$, por tanto, centradas en determinadas áreas de conocimiento. Estudios previos en el uso de la herramienta de exámenes de PoliformaT por parte de profesores de la Universidad Politécnica de Valencia (Vidal-Puig et. al., 2007; PortilloPoblador et. al., 2009; Vidal-Puig et. al., 2011 y Villa-Julia et. al., 2012) avalan el potencial del uso de esta herramienta, ahondando en las posibilidades actuales de la herramienta de exámenes de la plataforma educativa de la UPV. Finalmente, hemos optado por una estrategia mixta para la evaluación que utilice pruebas de tipo test opción múltiple en sus distintas modalidades (Norris y Ennis 1989) y pruebas tipo ensayo o informe de acuerdo con las habilidades o disposiciones consideradas en cada caso, siendo este último tipo de prueba el más apropiado para la evaluación de las habilidades más creativas asociadas al pensamiento crítico. Cada una de las actividades que se ha planteado ha sido evaluada y vinculada de acuerdo con los indicadores (entre los señalados en la Tabla 1) considerados al diseñar la actividad.

- Fase 4: Análisis del grado de consecución de las metas marcadas en las diferentes pruebas y actividades

Aunque en un primer momento el equipo se ha centrado más en desarrollar actividades y materiales, consideramos que se debe profundizar en el análisis y control de los resultados alcanzados; ya que esto nos permitirá reconsiderar algunos aspectos de la estrategia que habría que ir cambiando. Por todo lo anterior consideramos que, teniendo en cuenta la experiencia de este primer año, para los próximos cursos deberíamos mejorar los siguientes aspectos de la metodología:

- A la hora de planificar la asignatura hay que incorporar una tabla de especificaciones donde se dé la distribución de partes o pruebas que van a tener lugar y sus correspondientes ponderaciones en la nota final, de modo que se observe que la evaluación final del alumno cubre en proporción adecuada todos los indicadores de la competencia transversal considerados (descritos en la fase 2 y recogidos en la Tabla 1 ).

- La corrección, evaluación y comunicación de resultados en las diferentes pruebas y actividades realizadas por los alumnos deben realizarse dentro de unos márgenes adecuados de tiempo para que se potencie su aspecto formativo.

- La comunicación al alumno de sus resultados debe proveerles de comentarios personalizados o al menos darle comentarios generales, desde el punto de 
vista del pensamiento crítico, a todo lo observado por los profesores en la actividad realizada por los alumnos.

- La información obtenida en el propio proceso de corrección permitirá depurar la rúbricas específicas de corrección (especialmente en el caso de actividades de reciente creación).

\section{Resultados}

\subsection{Metodología y técnicas utilizadas para el tratamiento y análisis de los datos.}

Para analizar el grado de consecución de los objetivos prefijados se han utilizado los datos obtenidos a partir de tres tipos de evidencias clave: una encuesta de opinión del profesorado, una encuesta de opinión de los alumnos y los resultados obtenidos por los alumnos en las diferentes asignaturas. Para obtener la información a partir de los datos recogidos se han empleado diferentes técnicas de estadística descriptiva.

\subsection{Grado de consecución de los objetivos y resultados alcanzados}

La implantación de la metodología propuesta ha supuesto la creación de diferentes tipos materiales y actividades ya descritas en el punto 3, entre los que se citaban: actividades de análisis de un conjunto de datos sujetas a corrección por pares y discusión por parte de los alumnos haciendo uso de la herramienta tareas del PoliformaT; pruebas objetivas en sus diferentes versiones (pregunta de opción múltiple, preguntas de respuesta corta...) usando la herramienta Exámenes de PoliformaT; preguntas en las diferentes pruebas y tareas basadas en análisis de encuestas relacionadas con problemas contemporáneos.

Estos materiales creados sirven como evidencias de un nivel de logro adecuado en varios de los objetivos perseguidos en la innovación, tanto generales como específicos, que incluyen la propia elaboración de material docente, como se puede observar en la Figura 3.

En cuanto al objetivo general de estudio profundo de la competencia y la adopción de una definición de pensamiento crítico, se ha alcanzado un nivel adecuado de logro como evidencia toda la labor de documentación desarrollada por parte del profesorado implicado, la selección de indicadores, y la creación de material informativo para los alumnos (objetivo específico 1) que ha sido bien valorado por parte de estos, como pone de manifiesto la pregunta el resultado de la encuesta a los alumnos (Figura 5).

Respecto al tercero de los objetivos generales, que era conseguir la implantación de una metodología de evaluación continua y formativa en pensamiento crítico, las evidencias las basamos en tres elementos claves: la opinión del profesorado, la opinión de los alumnos y los resultados obtenidos por los alumnos.

- Opinión del profesorado

El profesorado implicado valoró en una encuesta (Figura 4) el grado de éxito de la metodología y la calidad de los materiales creados, obteniéndose un 6.69 y 7.75 sobre 10,

(cc) EY-NC-ND 2018, Universitat Politècnica de València

Congreso IN-RED (2018) 


\begin{tabular}{|c|c|c|c|}
\hline & Objetivo & Nivel & Evidencias \\
\hline \multirow{3}{*}{ 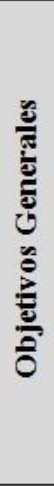 } & $\begin{array}{l}\text { A) Estudio profundo de la competencia y la } \\
\text { adopción de una definición de pensamiento } \\
\text { crítico }\end{array}$ & Adecuado & $\begin{array}{l}\text { - Selección de } \\
\text { indicadores } \\
\text { - Opinión de los } \\
\text { profesores } \\
\text { - Consecución objetivo } \\
\text { específico 1) }\end{array}$ \\
\hline & $\begin{array}{l}\text { B) Elaboración de material y preparación } \\
\text { de actividades }\end{array}$ & Adecuado & $\begin{array}{l}\text { Material creado: } \\
\text { consecución objetivos } \\
\text { específicos 2) 3) 4) 5) }\end{array}$ \\
\hline & $\begin{array}{l}\text { C) Implantación de una metodología de } \\
\text { evaluación continua y formativa en } \\
\text { pensamiento crítico }\end{array}$ & Adecuado & $\begin{array}{l}\text {-Opinión del } \\
\text { profesorado } \\
\text { - Opinión de los alumnos } \\
\text { - Resultados obtenidos } \\
\text { por los alumnos }\end{array}$ \\
\hline \multirow{7}{*}{ 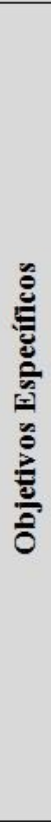 } & $\begin{array}{l}\text { 1) Preparación de material informativo para } \\
\text { los alumnos sobre la competencia } \\
\text { transversal }\end{array}$ & Adecuado & $\begin{array}{l}\text { - Documento explicativo } \\
\text { puesto a disposición de } \\
\text { los alumnos } \\
\text { - Resultados de pregunta } \\
\text { realizada en encuesta a } \\
\text { los alumnos }\end{array}$ \\
\hline & $\begin{array}{l}\text { 2) Preparación de material de formación en } \\
\text { pensamiento crítico para incluir en los } \\
\text { guiones de prácticas }\end{array}$ & $\begin{array}{c}\text { En } \\
\text { Progreso }\end{array}$ & Material creado \\
\hline & $\begin{array}{l}\text { 3) Preparación de preguntas para la } \\
\text { evaluación continua en pensamiento crítico } \\
\text { durante la sesiones de prácticas }\end{array}$ & Adecuado & $\begin{array}{l}\text { Material creado: } \\
\text { Preguntas y pruebas } \\
\text { poliformaT }\end{array}$ \\
\hline & $\begin{array}{l}\text { 4) Preparación de actividades individuales } \\
\text { o grupales para evaluar las habilidades más } \\
\text { creativas de pensamiento crítico }\end{array}$ & Adecuado & $\begin{array}{l}\text { Material creado: } \\
\text { Actividades diversas } \\
\text { creadas }\end{array}$ \\
\hline & $\begin{array}{l}\text { 5) Creación de pruebas y adaptación de las } \\
\text { rúbricas propuestas por el ICE para la } \\
\text { evaluación de la competencia en nuestra } \\
\text { materia específica }\end{array}$ & Adecuado & $\begin{array}{l}\text { Material creado: Rúbricas } \\
\text { individuales para cada } \\
\text { actividad }\end{array}$ \\
\hline & $\begin{array}{l}\text { 6) Estudio estadístico de los resultados } \\
\text { obtenidos para poder validar la } \\
\text { metodología empleada }\end{array}$ & Adecuado & $\begin{array}{l}\text { Estudio descriptivo de } \\
\text { resultados de las } \\
\text { encuestas }\end{array}$ \\
\hline & $\begin{array}{l}\text { 7) Comunicación de nuestros resultados a } \\
\text { la comunidad universitaria }\end{array}$ & Adecuado & $\begin{array}{l}2 \text { Congresos } \\
\text { internacionales }\end{array}$ \\
\hline
\end{tabular}

Fig 3 Nivel de consecución de los objetivos marcados

respectivamente. Como puntos fuertes se señalaron el conocimiento alcanzado por el profesorado en la competencia, cómo desarrollarla y evaluarla en los alumnos y la existencia de un profesorado muy implicado. Como puntos débiles se indicaron una cierta falta de tiempo y desbordamiento del profesor por las pruebas de evaluación (al menos en esta fase inicial de implantación).

- Opinión de los alumnos

La encuesta valora la satisfacción del alumnado con la metodología y actividades de formación y evaluación del pensamiento crítico. La Figura 5, donde se solicita la valoración sobre la información y documentación recibida respecto a la competencia de pensamiento 


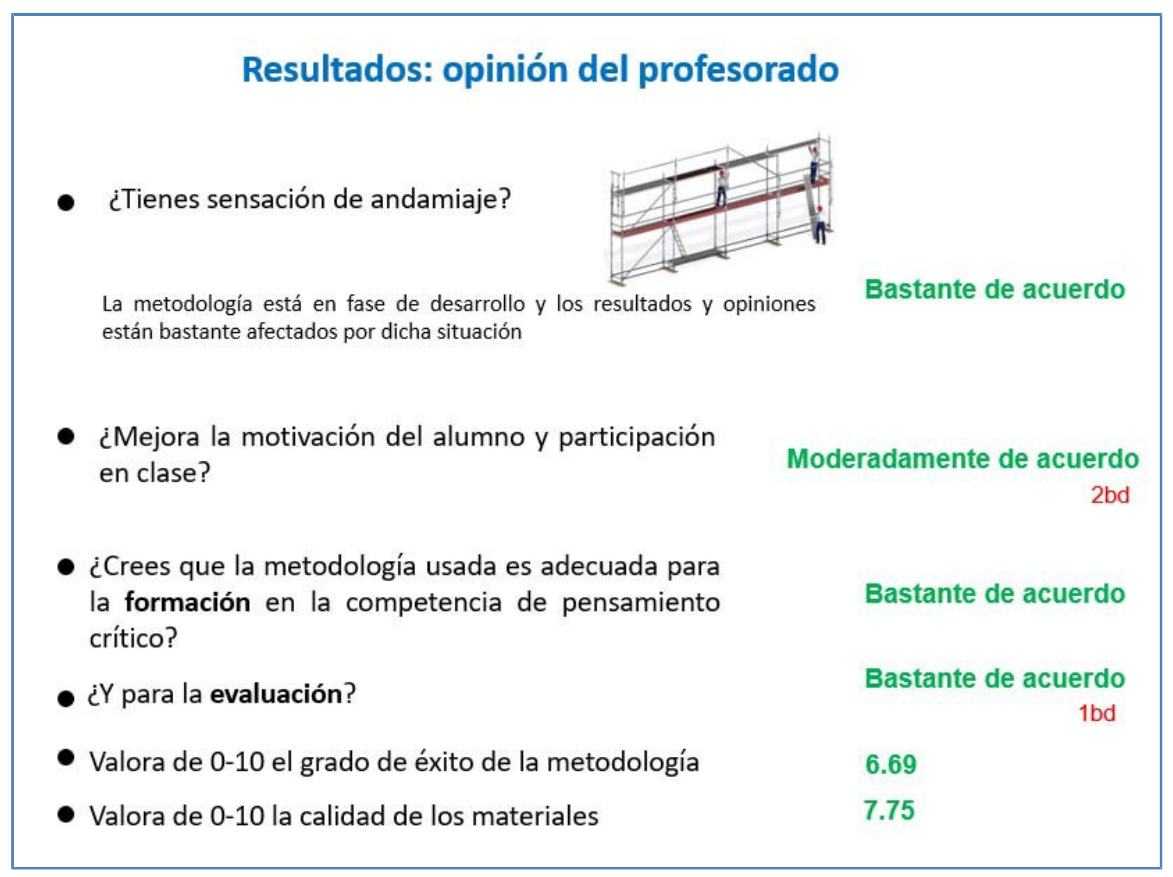

Fig 4: Opinión del profesorado sobre la metodología propuesta para la formación y evaluación en pensamiento crítico

crítico, muestra unos resultados favorables que constituyen una evidencia de la consecución de un nivel adecuado del objetivo general A y específico 1 dado el grado de satisfacción percibido por parte del alumnado.

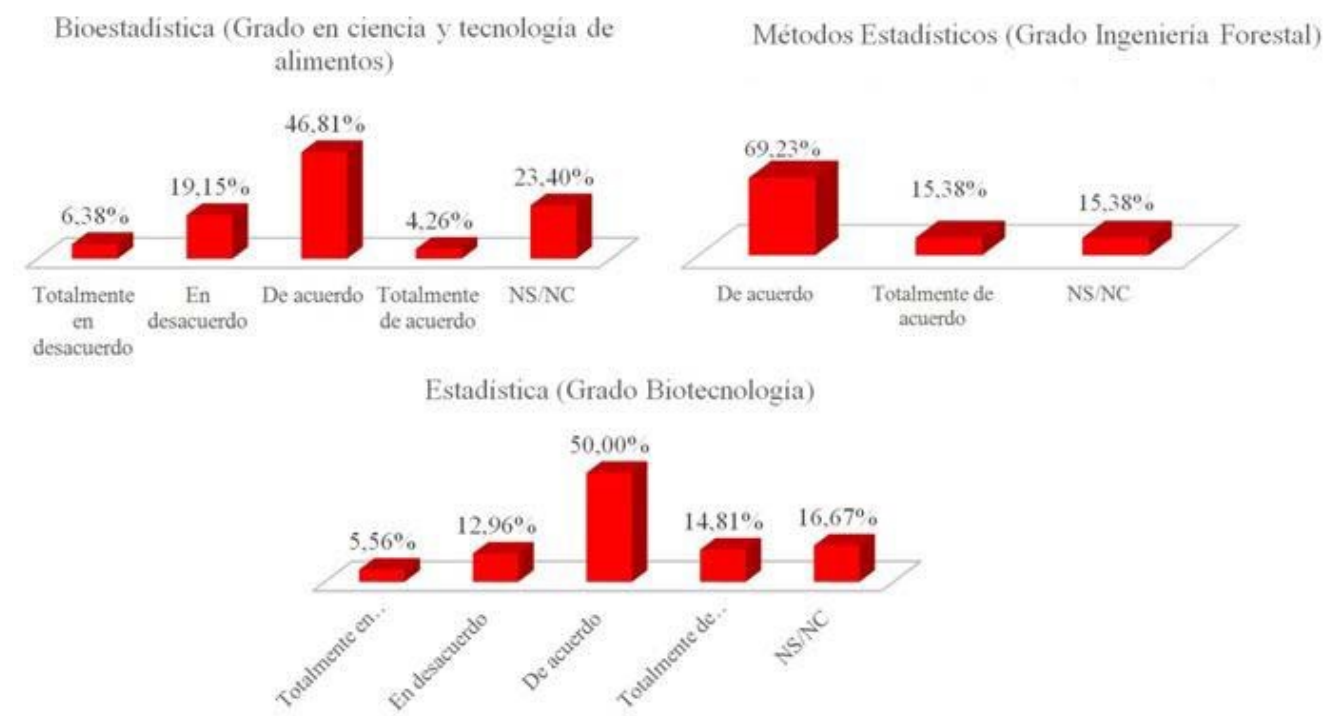

Fig 5: Opinión del alumnado sobre la información/documentación proporcionada sobre pensamiento crítico

(cc)) EY-NC-ND 2018, Universitat Politècnica de València

Congreso IN-RED (2018) 
Las Figuras 6 y 7 muestran la opinión del alumno sobre la metodología para la formación y evaluación propuesta. Los resultados son satisfactorios, pues las respuestas se agrupan mayoritariamente en un nivel medio-alto

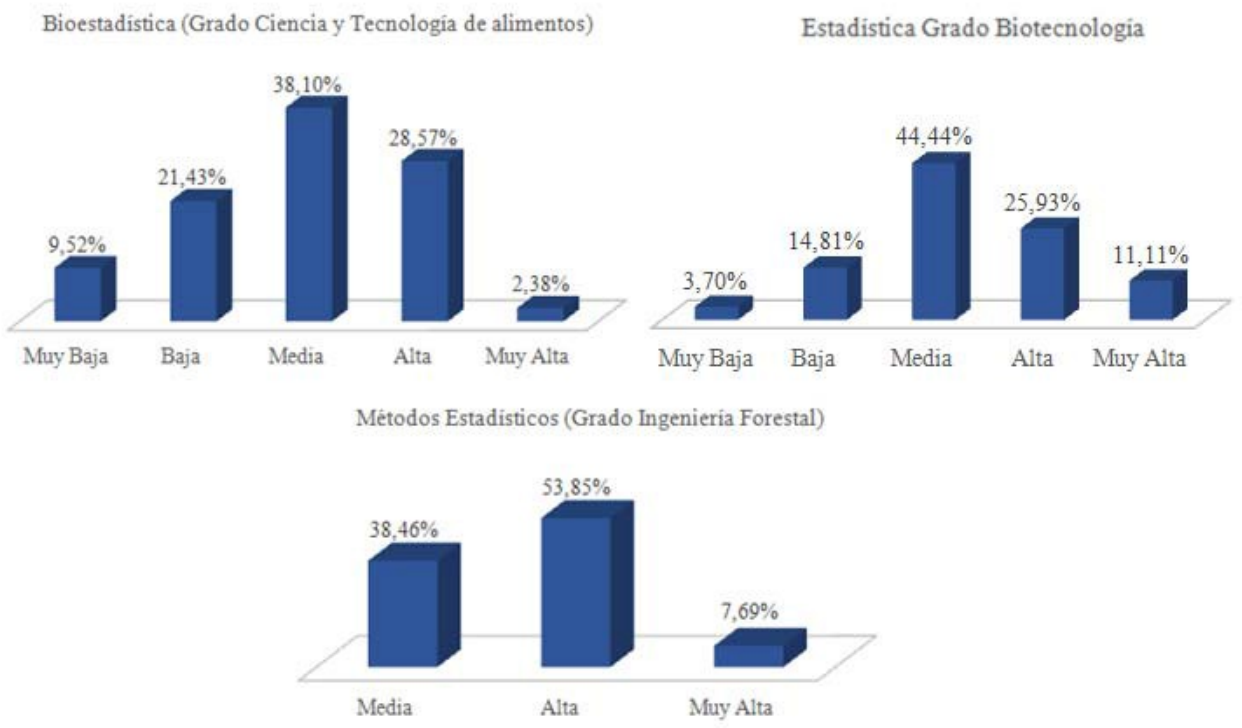

Figura 6: Opinión del alumnado sobre la metodología propuesta para la formación en pensamiento crítico

- Resultados de los alumnos

Los resultados de pensamiento crítico del estudiante varían entre titulaciones o grados, aunque hay algunos perfiles similares. Los estudiantes de los cursos de Ciencia y Tecnología de los Alimentos e Ingeniería Forestal son los grados donde mayor porcentaje de estudiantes alcanzan las calificaciones más altas, A y B. Además, estos grupos tienen los mismos perfiles de distribución de las calificaciones. Debido a que los grupos tienen un tamaño más pequeño fue posible una buena interacción estudiante-instructor. La alta coordinación entre los instructores y la retroalimentación rápida sobre la evaluación dada a los estudiantes ha sido clave para sus resultados.

Obviamente, las calificaciones no pueden ser las mismas en todos los grupos porque hay diversos factores que influyen en los resultados. El sistema de evaluación debería mejorarse para hacer una evaluación más precisa y consistente del pensamiento crítico. Hay grupos donde los resultados han sido poco satisfactorios. Sin embargo, reiteramos nuestra preocupación acerca de que mejorar el entrenamiento de los estudiantes en pensamiento crítico es esencial, pero también una retroalimentación temprana sobre el progreso del estudiante que mejore el aspecto formativo de esta metodología docente. Finalmente, queremos señalar que los sistemas de evaluación que hemos diseñado se han adaptado a grupos grandes haciendo uso de la evaluación por pares y de pruebas de corrección automática a través de la herramienta PoliformaT, que es la plataforma educativa en línea de la UPV que facilita la tarea del profesor y su comunicación con el alumnado. 

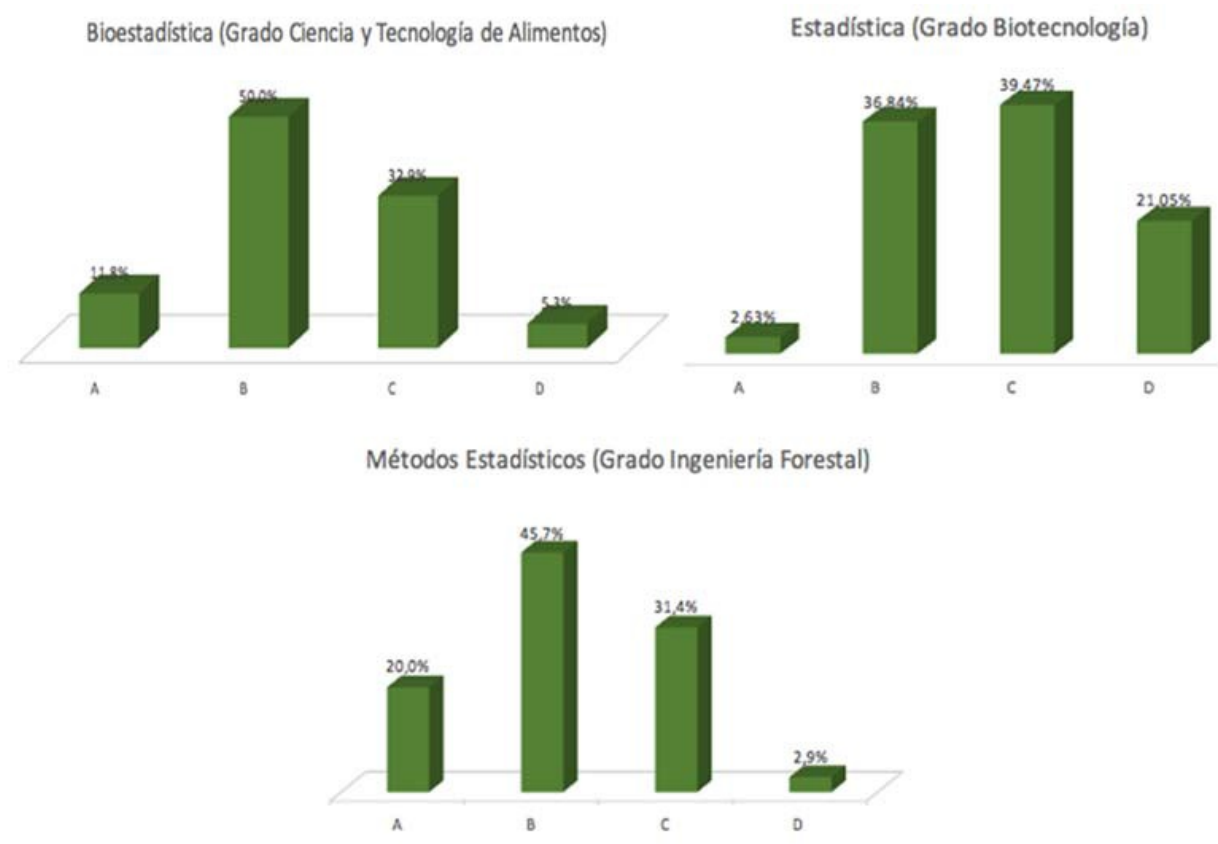

Figura 7: Resultados en la evaluación de los estudiantes del pensamiento crítico por titulación (A: Excelente; B: Adecuado; C: Desarrollandose; D: No alcanzado)

\section{Conclusiones}

Tras el primer año de implantación de la competencia del pensamiento crítico, hemos observado que se produce una retroalimentación entre el alumno y el profesor. Este hecho ayuda al docente en el perfeccionamiento y maduración de su pensamiento crítico. Por tanto, parece que este proceso lleva al docente a "enseñar aprendiendo".

La metodología en su primer año de aplicación ha mostrado una buena aceptación por parte del profesorado y del alumnado implicado, como lo han puesto de manifiesto las diferentes encuestas y los resultados obtenidos por los alumnos. Ha sido una experiencia interesante, aunque todavía está en fase de desarrollo e implantación.

Uno de los resultados importantes es que el profesorado implicado ha mejorado su aptitud para la creación de nuevos materiales y metodologías que incorporen la formación y evaluación en pensamiento crítico y lo ha incorporado a su modo habitual de trabajo. Como grupo somos conscientes de que este proyecto es complejo y debe estar sometido a continuo debate y mejora.

Otro de los aspectos a considerar es el de lograr una mejor integración en la evaluación final del alumno de un conjunto de evidencias procedentes de diferentes pruebas de naturaleza cualitativa y cuantitativa u otros aspectos relacionados con la metaevaluación.

También sería interesante la creación de herramientas estadísticas apropiadas que faciliten el seguimiento de los alumnos y permitan facilitar la retroalimentación.

(cc) EY-NC-ND 2018, Universitat Politècnica de València

Congreso IN-RED (2018) 


\section{Referencias}

AIZIKOVITSH-UDI, E., KUNTZE S., CLARKE, D. (2016). "Connections Between Statistical Thinking and Critical Thinking: A Case Study“ In: Ben-Zvi D., Makar K. (eds) The Teaching and Learning of Statistics. Switzerland : Springer International Publishing, pp 83-94.DOI.10.1007/978-3319-23470-0_8

ENNIS, R. H. (1993). Critical thinking assessment. Theory into Practice, vol. 32 issue 3, p. 179-186

NORRIS, S. P. y ENNIS, R. H. (1989). Evaluating critical thinking. Pacific Grove, CA: Midwest Publications

PORTILLO-POBLADOR, N. et al. (2009). "E-learning in teaching statistics: an application in civil engineering” en EDULEARN09 1st International Conference on Education and New Learning Technologies. (6-8 Julio 2009. Barcelona)

VIDAL-PUIG, S. et al. (2007).“New methodology for teaching statistics which allows the progressive assessment of students". International Association of Technology, Education and Development en INTED2007 (7-9 Marzo 2007. Valencia)

VIDAL-PUIG, S. et al. (2011)."Educational innovation project in teaching statistics in engineering degrees" 4th International Conference of Education, Research and Innovation en ICERI2011 (14-16 November 2011. Madrid)

VILLA-JULIA, M.F. et al.(2012) “Educational innovation project in teaching statistics in engineering degrees II“ 5th International Conference of Education, Research and Innovation en ICERI2012 (1921 November 2012. Madrid)

INSTITUTO DE CIENCIAS DE LA EDUCACIÓN UPV. Documento informativo sobre el proyecto institucional de Competencias Transversales UPV

http://www.upv.es/entidades/ICE/info/U0724624.pdf 